Meta

Journal des traducteurs

Translators' Journal

\title{
Un projet terminologique : l'observatoire de néologismes scientifiques et techniques du portugais du Brésil
}

\section{Ieda Maria Alves}

Volume 41, numéro 2, juin 1996

Traduction et terminologie au Brésil

Translation and Terminology in Brazil

URI : https://id.erudit.org/iderudit/001880ar

DOI : https://doi.org/10.7202/001880ar

Aller au sommaire du numéro

Éditeur(s)

Les Presses de l'Université de Montréal

ISSN

0026-0452 (imprimé)

1492-1421 (numérique)

Découvrir la revue

Citer cet article

Alves, I. M. (1996). Un projet terminologique : l'observatoire de néologismes scientifiques et techniques du portugais du Brésil. Meta, 41(2), 255-258. https://doi.org/10.7202/001880ar
Résumé de l'article

Cet article présente un projet de recherche sur les néologismes techniques et scientifiques en portugais du Brésil. On présente la méthodologie du projet, ses bases théoriques, ses objectifs ainsi que les caractéristiques morphologiques générales des néologismes recueillis. 


\title{
UN PROJET TERMINOLOGIQUE: L'OBSERVATOIRE DE NÉOLOGISMES SCIENTIFIQUES ET TECHNIQUES DU PORTUGAIS DU BRÉSIL
}

\author{
IEDA MARIA ALVES
}

Université de São Paulo, São Paulo, Brésil

\begin{abstract}
Résumé
Cet article présente un projet de recherche sur les néologismes techniques et scientifiques en portugais du Brésil. On présente la méthodologie du projet, ses bases théoriques, ses objectifs ainsi que les caractéristiques morphologiques générales des néologismes recueillis.
\end{abstract}

Abstracts

This article will present a research project on technical and scientific neologisms in Brazilian Portuguese. It presents the project's methodology, the theorerical framework, the objectives, and specifies the general morphological features of the neologisms collected.

Le dépouillement systématique de néologismes dans la presse brésilienne, que nous effectuons depuis plusieurs années, nous a appris que le phénomène de la néologie lexicale est beaucoup plus intense dans des domaines spécialisés des sciences et des techniques, les langues de spécialité, que dans la langue courante.

La politique brésilienne, si instable depuis le coup d'État de 1964, constitue une source inépuisable de créativité lexicale. À chaque élection, à chaque changement de ministère, le langage joue un rôle important, en tant que reflet de la transformation que subit le pays. Les changements politiques ont, sans conteste, une influence sur l'économie du pays qui, elle aussi, constitue un miroir de toutes les transformations sociales et budgétaires vécues par le peuple brésilien.

Les changements et les difficultés qui caractérisent la vie des Brésiliens dans les dernières décennies n'empêchent pas, heureusement, que les sciences et les techniques progressent dans le pays. Comme partout, le phénomène de la néologie lexicale - ou néonymie, selon la terminologie propre à Rondeau (1984: 121) — est très productif dans les divers domaines scientifiques et techniques et montre, par conséquent, un développement scientifique et technique au Brésil.

Malgré ce progrès, la quasi-inexistence d'études néonymiques de caractère systématique dans le portugais du Brésil rend impossible que des chercheurs, traducteurs ou professionnels dénomment, de façon plus adéquate, les concepts créés par les besoins des sciences et des techniques.

Pour cette raison, nous développons depuis 1988 un projet terminologique ayant l'objectif de décrire, de façon systématique, la néologie lexicale dans quelques domaines des langues de spécialité. Ce projet vise, par conséquent, à concilier le progrès dans quelques domaines des sciences et des techniques avec la créativité lexicale correspondante.

Dans cet exposé, nous décrirons, premièrement, les procédés méthodologiques que nous avons adoptés. Dans une deuxième partie, et en nous basant sur les néonymes 
dépouillés, nous exposerons les caractères propres des formations néonymiques du portugais du Brésil.

\section{MÉTHODOLOGIE}

L'Observatoire se propose de dépouiller, d'analyser et de diffuser des aspects de la néologie technique et scientifique du portugais brésilien.

Parmi les diverses sciences et techniques, nous avons privilégié, premièrement, les domaines de l'économie, de l'informatique, des sciences agraires, de la psychologie et du tourisme. L'économie et l'informatique, en particulier, constituent une source de créativité néonymique. Dans le domaine de l'informatique, la créativité est due, certainement, au développement croissant de cette science, et en ce qui concerne l'économie, nous considérons que ce fait est causé par les changements subis par les Brésiliens dans les dernières décennies.

Le dépouillement des néonymes est effectué par des étudiants en lettres, qui reçoivent des bourses du Conselho Nacional de Desenvolvimento Científico $e$ Tecnológico $(\mathrm{CNPq})$ et de la Fundação de Amparo à Pesquisa no Estado de Sáo Paulo (FAPESP). Ces étudiants collectent des termes dans deux types de documents : des revues spécialisées dans les domaines étudiés et des revues et des cahiers de journaux de vulgarisation scientifique et technique. Ce double caractère du corpus nous permet d'avoir, au moyen des revues spécialisées, un corpus technique et, en plus, un corpus de divulgation destiné à des non-spécialistes. On peut vérifier, par conséquent, le mouvement néonymique, spécialisé et de divulgation, relatif à un domaine.

Le corpus d'exclusion qui nous sert de support pour la reconnaissance des néonymes est constitué, dans chaque domaine, par des dictionnaires spécialisés. Le Novo Dicionário da Língua Portuguesa, de Holanda (1986), en tant que dictionnaire de langue le plus diffusé et le plus employé au Brésil, fait aussi partie du corpus d'exclusion pour tous les domaines étudiés.

Les néonymes dépouillés sont transcrits dans une fiche terminologique informatisée, qui comprend les champs suivants concernant le néonyme collecté: le terme; le sigle ou les formes abrégées; les références grammaticales; le(s) contexte(s); les références du(des) contexte(s); la définition; le domaine; le sous-domaine; les notes linguistiques; les notes encyclopédiques; le(s) synonyme(s); le(s) terme(s) connexe(s); l'auteur de la fiche; le réviseur de la fiche; la date d'enregistrement.

Le projet «Observatoire» compte, actuellement, près de 8000 termes, ainsi distribués d'après les domaines: psychologie - près de 900 termes; tourisme - près de 500 termes; informatique — près de 2000 termes; sciences agraires — près de 3500 termes; économie - près de 700 termes.

\section{CARACTÉRISTIQUES MORPHOLOGIQUES DES NÉONYMES}

La collecte systématique de termes dans le cadre du projet «Observatoire» nous permet de vérifier les caractéristiques dont se revêtent les formations néonymiques $d u$ portugais contemporain du Brésil.

Les procédés de formation lexicale tels que la dérivation, la composition, les formations acronymiques et syntagmatiques, la troncation, le transfert sémantique et les emprunts sont également présents dans la langue commune et dans les langues de spécialité. Malgré ces traits communs, les langues de spécialité privilégient quelques mécanismes de formation lexicale et révèlent, de cette manière, quelques caractéristiques par rapport à l'ensemble lexical du portugais. 
D'après l'analyse des néonymes déjà dépouillés dans les domaines analysés du projet «Observatoire», nous pouvons constater que les formations syntagmatiques - ou les termes-syntagmes d'après Kocourek ${ }^{1}$ (1982:116) - constituent le mode de formation le plus productif dans tous les domaines analysés.

Ce fait n'est pas exclusif au portugais du Brésil. Il a déjà été signalé par rapport au portugais européen et à d'autres langues latines 2 (voir Boulanger (1988) et Portelance (1989)). Le caractère onomasiologique de la terminologie tend à favoriser ce type de formation, car les termes-syntagmes constituent des créations presque toujours motivées qui, dans la majorité des cas, expriment leurs concepts respectifs de manière transparente.

Au cours de cet exposé, les exemples que nous présentons appartiennent au domaine de l'économie.

Dans ce domaine, les termes-syntagmes présentent différentes modalités de formation. Introduits par un nom, élément déterminé, ils sont suivis d'une expansion représentée par un ou plusieurs déterminants. Parmi ces déterminants, les modalités les plus usuelles sont celles constituées par un adjectif : aceleração inflacionária, cesta básica, demanda agregada, espiral inflacionária; ou par un syntagme prépositionnel: aceleração dos preços, bolha de consumo.

D'autres formes d'expansion, plus complexes, sont présentes dans le domaine, telles que:

adjectif + adjectif : demanda agregada nominal, receita tributária bruta;

adjectif + syntagme prépositionnel: crescimento sazonal da economia, emissão passiva da moeda, expansão nominal da moeda, mercado internacional de capitais;

syntagme prépositionnel + une autre expansion: bens de consumo durável, bens de consumo não-durável, programa de ajustamento econômico, taxa de câmbio de equilíbrio, taxa de juros reais positivos.

Les formations syntagmatiques, très souvent, sont employées sous forme acronymique dans le domaine de l'économie. Elles dénominent, dans ce cas, des impôts, des indicatifs de salaires et de prix, des applications financières. Quelques exemples: CDB (Certificado de depósito bancário), DER (Depósito especial remunerado), FAS (Fator de atualização salarial), IGP (Índice geral de preços), INA (Indicador de nível de atividades), INC (Indicador de nivel de consumo), INC (Índice nacional de preços ao consumidor), IOF (Imposto sobre operaçōes financeiras), TRD (Taxa referencial diária), VBC (Valores básicos de custeio). Quelques sigles font aussi partie d'un syntagme, lorsqu'ils se présentent accompagnés d'un adjectif : PIB agrícola, PIB industrial.

La plupart des sigles du domaine analysé sont formés d'au moins deux lettres initiales du syntagme. Quelques-uns, comme Ibovespa (Índice da Bolsa de Valores de São Paulo) et Mercosul (Mercado Comum do Sul), sont constitués de lettres et de groupes de lettres initiales. Cette catégorie de sigles est appelée, par Germain et Lapierre (1988: 56), sigles à lettres initiales, et se distingue du sigle acronyme, formé par des lettres ou des groupes de lettres initiales et finales.

Du point de vue de la dérivation, les néonymes du domaine de l'économie privilégient certains préfixes et certains suffixes. Le préfixe des-, très fréquent, marque la chute ou la décroissance d'une activité financière (desabastecimento, deságio, descongelamento, desinflação), tandis que des suffixes qui marquent une action, comme -ção et -mento, sont souvent employés pour dénoter un procès en développement: dolarização, remonetização, terceirização, enxugamento). L'excès est marqué soit par des préfixes mega-, sobre- (megadéficit, sobreendividamento, sobrepreço), soit par les suffixes -ão et -aço: fundão, mensalão, tarifaço. 
Le procès de la composition est représenté par plusieurs unités neónymiques, formées, très souvent, par deux noms : dólar-turismo, moeda-mercadoria, salário-educação, seguro-saúde.

D'autres mécanismes de formations néonymiques, moins productifs, sont aussi présents dans le domaine de l'économie, tels que la troncation (estagflação - estagnação + inflação; instagflação - instabilidade + estagnação + inflação), le transfert sémantique (desaquecimento, enxugamento). Les emprunts à l'anglais, si fréquents dans la terminologie de l'économie du portugais du Brésil, ne produisent, de nos jours, que des néonymes sporadiques: commodities agricolas, operaçöes de hedge.

\section{NOTES FINALES}

Ces néonymes dépouillés sont voués à la diffusion et, d'après ce principe, ils peuvent être consultés et utilisés (dans des articles, des thèses...) par le public intéressé. Des accords internationaux, établis en fonction de ce projet par l'Université de São Paulo avec des organismes tels que le Centre de Terminologie et de Néologie de 1'Institut de la Langue Française (Paris, France) et le Département d'études linguistiques de l'Université nouvelle de Lisbonne (Lisbonne, Portugal) prévoient la diffusion et l'utilisation du matériel collecté.

\section{Notes}

1. Étant donné le grand nombre de désignations pour le concept de syntagme lexical - Kocourek (p. 117) en cite vingt-sept - , nous les dénommerons des formations syntagmatiques ou des termes-syntagmes.

2. Tous les travaux réalisés par les membres de l'Association de Terminologie Portugaise (Termip), à Lisbonne, attestent ce phénomène.

\section{RÉFÉRENCES}

BOULANGER, J.-C. (1988) : «Le syntagme terminologique : projet de recherche», Terminogramme, 46.

GERMAIN, C. et A. LAPIERRE (1988): «Le sigle. Définition, caractéristiques et emploi», Cahiers de lexicologie, 53, pp. 51-68.

HOLANDA, Aurélio Buarque de (1986): Novo Dicionário da Lingua Portuguesa, Rio de Janeiro, Nova Fronteira.

KOCOUREK, R. (1982): La langue française de la technique et de la science, Paris, La Documentation française.

PORTELANCE, C. (1989) : «Syntagme et paradigme», Meta, 34-3, pp. 398-404.

RONDEAU, Guy (1984): Introduction à la terminologie, $2^{\circ}$ édition, Québec, Gaétan Morin Éditeur. 